\title{
Semiautomated object-based classification of rain-induced landslides with VHR multispectral images on Madeira Island
}

\author{
Sandra Heleno, Magda Matias, Pedro Pina, and António Jorge Sousa \\ CERENA, Instituto Superior Técnico, University of Lisbon, Lisbon, Portugal \\ Correspondence to: Sandra Heleno (sandra.heleno@ist.utl.pt)
}

Received: 30 July 2015 - Published in Nat. Hazards Earth Syst. Sci. Discuss.: 23 September 2015

Revised: 12 April 2016 - Accepted: 15 April 2016 - Published: 26 April 2016

\begin{abstract}
A method for semiautomated landslide detection and mapping, with the ability to separate source and run-out areas, is presented in this paper. It combines object-based image analysis and a support vector machine classifier and is tested using a GeoEye-1 multispectral image, sensed 3 days after a major damaging landslide event that occurred on Madeira Island (20 February 2010), and a pre-event lidar digital terrain model. The testing is developed in a $15 \mathrm{~km}^{2}$ wide study area, where $95 \%$ of the number of landslides scars are detected by this supervised approach. The classifier presents a good performance in the delineation of the overall landslide area, with commission errors below $26 \%$ and omission errors below $24 \%$. In addition, fair results are achieved in the separation of the source from the run-out landslide areas, although in less illuminated slopes this discrimination is less effective than in sunnier, east-facing slopes.
\end{abstract}

\section{Introduction}

Landslides are complex mass movements that occur on hill slopes due to the action of gravity; they play an important role in the evolution of landforms, while constituting a serious natural hazard in many regions throughout the world. Landslides can involve flowing, sliding, toppling, or falling and are commonly associated with a trigger: slope failures generally occur within minutes after an earthquake, hours to days after a snowmelt, and days to weeks after an intense rainfall (Guzzetti et al., 2012; Malamud et al., 2004). Urban expansion into hilly or mountainous regions results in more people being exposed to the hazard, thus increasing landslide risk. Nowadays, landslides claim thousands of lives every year and result in extensive infrastructure and prop- erty damage (Malamud et al., 2004; Yang and Chen, 2010; Holbling et al., 2012). Landslide susceptibility and hazard assessment are important tools in land-use planning, in particular to avoid urban expansion into vulnerable areas, thus reducing future economic and human losses. Past landslides are one of the best indicators of future landslide activity, and mapping landslides is therefore an essential step in hazard assessment (Bucknam et al., 2001; Lahousse et al., 2011; Aksoy et al., 2012; Guzzetti et al., 2012).

\subsection{Landslide inventories}

An inventory map records the location and, when known, the date of occurrence and type of landslides that have left discernible traces in an area (Malamud et al., 2004). A landslide-event inventory consists of all the slope failures associated with a single trigger such as an earthquake, rainstorm, or snowmelt and is useful to determine the residual risk in the aftermath of the event, as a guide for emergency and recovery efforts, and to validate landslide susceptibility and hazard models (Malamud et al., 2004; Barlow et al., 2006; Guzzetti et al., 2012; Mondini et al., 2013, 2014). Immediately after the event, individual landslides are easy to recognize, even in the case of small and shallow landslides such as soil slips or debris flows, and detailed mapping carried out shortly after the landslide event leads in general to a significantly complete inventory (Malamud et al., 2004). This notwithstanding, landslide inventories are generally incomplete, both in what concerns the area covered and the time period investigated, a serious drawback for landslide hazard studies (Malamud et al., 2004; Van Westen et al., 2006). 
Landslide inventories traditionally used to be derived by visual interpretation of aerial photographs and field surveys. The latter can lead to comprehensive and precise landslide inventories, but they are often hindered by cost effectiveness and logistical constraints, particularly over large or remote areas (Yang and Chen, 2010). Interpretation of stereoscopic aerial photography (whereby the interpreter detects and classifies landslides based on experience and on the analysis of changes in the form, shape, position, or appearance of the topographic surface) remains a common method to recognize landslides, despite being an empirical and subjective technique (Guzzetti et al., 2012). Aerial photographs, which can accurately depict the distribution and contours of landslides in a region, are seldom available in a timely manner, thus restricting the ability to prepare event and seasonal inventory maps repeatedly and for large areas (Martha et al., 2010; Aksoy et al., 2012; Guzzetti et al., 2012). In this context, and if the persistence of cloud cover is not an issue, satellite imagery emerges as a uniquely reliable tool for timely mapping of landslides and damage assessment over large and inaccessible areas (Barlow et al., 2006; Joyce et al., 2008; Aksoy et al., 2012; Holbling et al., 2012; Xu et al., 2015).

\subsection{Automated methods for landslide mapping}

Visual interpretation of satellite imagery remains extremely demanding in terms of resources and time, especially when dealing with numerous multi-scale landslides affecting wide areas, such as rainfall-induced shallow landslides. Applying automated methods can contribute to more efficient landslide mapping and updating of existing inventories, and in recent years the number and variety of approaches has rapidly increased (Guzzetti et al., 2012; Holbling et al., 2012; Marc and Hovius, 2015). Landslides can display highly heterogeneous sizes, demanding information with higher spatial resolutions in order to produce complete event inventories. Very highresolution (VHR) multispectral images, acquired by spaceborne sensors with sub-metric precision, such as Ikonos, QuickBird, GeoEye, or WorldView, are increasingly considered the best option for landslide mapping (Van Westen et al., 2008), but these new levels of spatial detail present new challenges to state-of-the-art image analysis tools (Kurtz et al., 2014).

In recent years, several semiautomated methods have been developed to tackle such difficulties, using specific classification schemes that target single post-event optical images (Cheng et al., 2013; Moosavi et al., 2014) or, when suitable pre-event data are available, exploit pre- and post-event image changes (e.g., Lu et al., 2011; Mondini et al., 2011a, b). In the latter case, great care has to be taken in the coregistration and radiometric correction procedures. Ideally, pre-event and post-event images should be acquired at the same time of the year and with similar view angle and solar illumination, but this is often not feasible (Guzzetti et al., 2012).
Semiautomated approaches to landslide mapping can be classed, according to the type of image element used, as "pixel based" (e.g., Chang et al., 2007; Yang and Chen, 2010; Chini et al., 2011; Cheng et al., 2013; Mondini et al., 2013, 2011a, b) or "object based" (e.g., Aksoy et al., 2012; Holbling et al., 2012, 2015; Lacroix et al., 2013; Lahousse et al., 2011; Lu et al., 2011; Martha et al., 2010, 2011, 2012, 2013; Stumpf et al., 2011, 2014; Van Den Eeckhaut et al., 2012). When applied to very high spatial resolution images, pixel-based methods often exhibit a "salt and pepper" appearance (Van Westen et al., 2008; Guzzetti et al., 2012) which requires image post-processing. The "object-oriented" approach, however, groups image pixels into homogeneous objects, with shape, size, neighboring, and textural features in addition to spectral information (Aksoy et al., 2012). With both approaches, supervised and unsupervised classification schemes have been adopted, based on algorithms such as maximum likelihood (Nichol et al., 2005; Borghuis et al., 2007; Danneels et al., 2007), $K$ nearest neighbor (Cheng et al., 2013; Li et al., 2013), artificial neural networks (Nichol et al., 2005; Danneels et al., 2007; Moosavi et al., 2014), random forests (Stumpf et al., 2011, 2014), or support vector machines (SVMs; Pisani et al., 2012; Van Den Eeckhaut et al., 2012; Moosavi et al., 2014). Novel object-based approaches for automated landslide mapping include the classification of different landslide types (Martha et al., 2010), identification of landslides from panchromatic imagery only through strong reliance on texture measures (Martha et al., 2012), or the detection and mapping of forested landslides resorting to lidar data (Van Den Eeckhaut et al., 2012).

The separation of the landslide-affected region into source, transport, and deposition areas is important to support postevent mitigation actions, since sediments deposited by landslides are likely to become source materials in subsequent events (Mondini et al., 2011a; Lira et al., 2013). More generally, the assessment of the volume of sediments produced, displaced, and deposited by landslides is important for susceptibility and hazard evaluation (Guzzetti et al., 2009). Mondini et al. (2011a, 2013) developed semiautomated pixel-based approaches to map landslides into source and run-out areas (defined as the union of transport and depositional areas), using a single post-event image and pre- and post-event image changes, respectively. Recently, Holbling et al. (2015) developed an object-based approach for semiautomated landslide change detection, with the ability to separate landslide sources from debris flow/sediment transport areas. Van Den Eeckhaut et al. (2012) also separate landslide source area and run-out but, in contrast to the previous authors, they use lidar data instead of optical imagery.

\subsection{Layout of the present work}

In this work we develop and test a methodology for semiautomated landslide recognition and mapping of landslide source and run-out areas. The method combines object-based image 


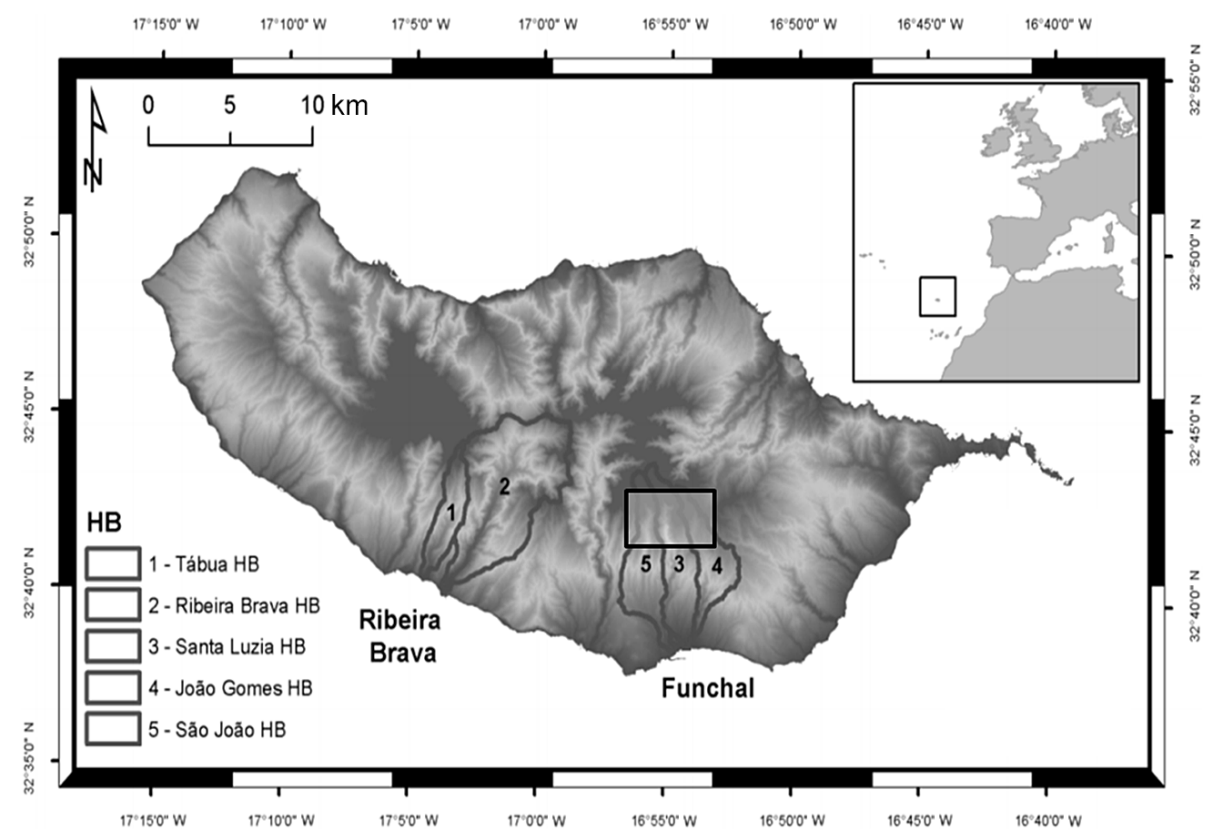

Figure 1. Location of Madeira Island, in the North Atlantic, with the most affected basins during the 2010 event superimposed on a DTM. Our study area is delimited by the rectangle located over Funchal basins. HB: hydrographic basin. Adapted from Lira et al. (2013).

analysis and an SVM supervised learning algorithm, and it is tested with information from VHR multispectral imagery acquired after a landslide event, together with a pre-event high-resolution $(4 \mathrm{~m})$ lidar digital terrain model (DTM). Our motivation to develop a procedure targeting post-event images solely, instead of change detection approaches, stems from the extreme difficulties often experienced in the coregistration of pre-event and post-event images in very steep regions (Pollard et al., 2010). Madeira Island, where our method was tested, is an example of a region where accurate co-registration was not possible (Chini et al., 2011). We test the approach on a GeoEye- 1 multispectral image $(0.5 \mathrm{~m}$ panchromatic band) acquired 3 days after an exceptionally heavy rainfall episode in February 2010 that triggered thousands of shallow landslides and flash floods across Madeira Island, in the North Atlantic, and claimed 45 lives (Lira et al., 2013). Section 2 describes the imagery and ancillary data used in the study and the preparation of a landslide inventory to evaluate the results. Section 3 presents the procedures for semiautomated landslide mapping and quality assessment. The results are presented in Sect. 4, followed by a brief discussion and conclusion in Sect. 5.

\section{Data}

\subsection{Study area and imagery data sets}

Madeira Island (Fig. 1), with a population of 250000 inhabitants, has a long record of flash floods, with at least 30 flash flood events of significant intensity registered since the be- ginning of the 19th century (Baioni, 2011; Lira et al., 2013). These flash floods usually last a few hours, during which a large amount of sediment is transported downstream very rapidly and with very high energy. Large part of the transported material is sourced by shallow landslides triggered upstream by the heavy precipitation (Lira et al., 2013). The combination of rough and steep terrain (mean slope angle $37 \%$ ) with intense rainfall provides the conditions for frequent and widespread landslides on Madeira.

On 20 February 2010 an extreme rainfall event followed a prolonged precipitation period (Luna et al., 2011; Couto et al., 2012; Fragoso et al., 2012; Teixeira et al., 2014). In the first hours of the morning rainfall values reached more than the double of the monthly average, triggering landslides and exceptionally strong flash floods that affected severely the municipalities of Funchal (home to half of the island population) and Ribeira Brava (Lira et al., 2013).

Our study area $\left(15 \mathrm{~km}^{2}\right.$ area rectangle in Fig. 1) covers one of the regions most affected by the 2010 landslide event on Madeira, in the south sector of the island, enclosing the main drainage basins upstream from the city of Funchal. Three days after the disaster, the GeoEye-1 satellite was programmed, in the scope of the GMES SAFER project, to acquire images over Madeira. The post-event image (Fig. 2) comprises four spectral bands (R, G, B, and near-infrared, or NIR) with $2.0 \mathrm{~m}$ spatial resolution and one panchromatic band with $0.5 \mathrm{~m}$ spatial resolution. Figure $2 \mathrm{~b}$ shows the sediments transported downhill by the flood, flowing into the ocean in Funchal downtown area. Ancillary data used in this study include pre-event (2007) and post-event (May 2010) 
(a)

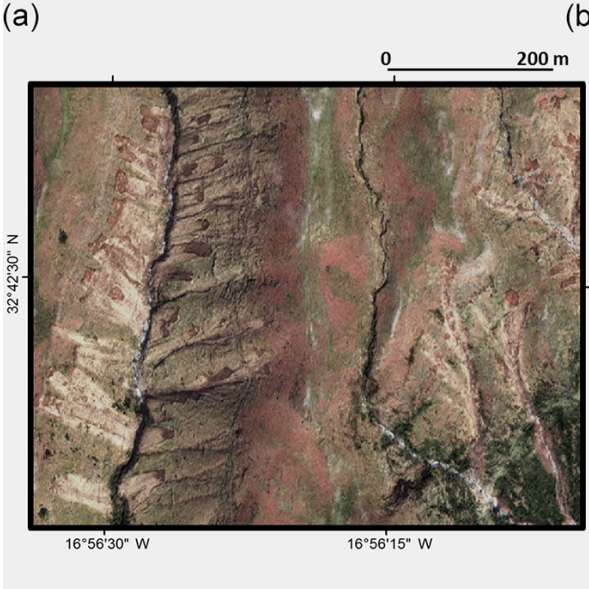

(b)

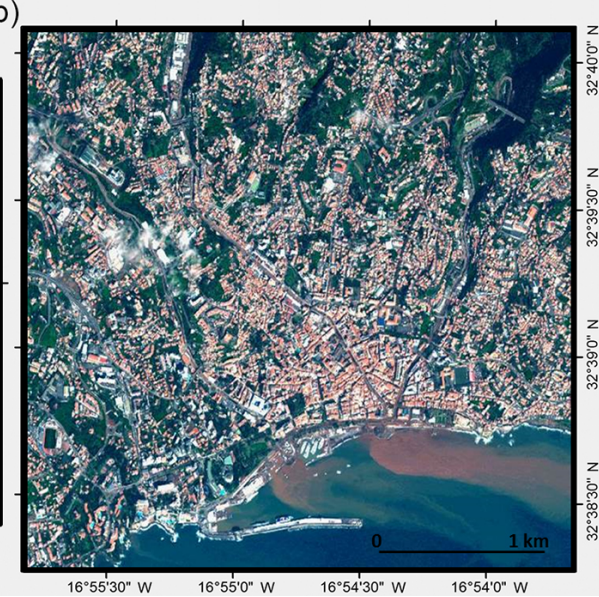

Figure 2. Details of GeoEye-1 pan-sharpened RGB image acquired over Madeira 3 days after the flash floods.

orthophotos acquired by airplane, with $0.4 \mathrm{~m}$ resolution, and a lidar pre-event DTM with $4 \mathrm{~m}$ resolution. The lidar data were acquired in 2009 with a Leica ALS50 airborne mapping system, with original point density $1 \mathrm{~m}^{-2}$.

\subsection{Landslide inventory}

A landslide reference data set was prepared to train and evaluate the semiautomated method, by thorough revision of a previous inventory produced by Lira et al. (2013), based on delimitation of contours on the post-event GeoEye-1 image and assisted by visual interpretation of the orthophotos. After the revision of its contours, the landslides were classified according to the type of movement (Varnes, 1978; Cruden and Varnes, 1996; Zêzere, 2002), resorting to several data sources besides the imagery, namely field work reports and photographic evidence. The large majority of mass movements were shallow translational slides and debris flows. The latter are the fastest and most destructive type of landslide and important sources of sediment to channel networks; most begin as translational slides that liquefy (Gabet and Mudd, 2006; Sidle and Ochiai, 2006). For the drainage basins of the Funchal and Ribeira Brava municipalities, 3207 shallow translational slides, 795 debris flows, and 59 rotational slides were inventoried. Furthermore, source and run-out areas were mapped separately inside the disturbed region. In the case of shallow translational slides, the separation was based on the darker appearance of the source areas (corresponding to freshly uncovered deep soil) which contrasted with the brighter run-out areas characterized by disturbed and/or bent vegetation and superficial layers of soil. For the debris flow tracks, darker, fresher-looking areas were interpreted as scoured sectors acting as important sources of material. This is expected to occur in the steepest sectors of the drainage network (e.g., Schurch et al., 2011; Theule et al., 2014; Tiranti et al., 2015), which is the case of our study area. The distinction between source and run-out areas was easier for fresh translational slides than for debris flows, but in both cases the process was affected by considerable uncertainty. Given the absence of anomalous precipitation in the months following the event (IPMA, 2010), we were able to minimize the mapping errors to some extent by re-interpreting the same landslides on the orthophotos acquired a few months later (May, 2010), when all loose material had been washed away from run-out areas. Often the landslides are composed of a shallow translational slide that further develops into debris flows (as reported and illustrated for Madeira Island in Lira et al., 2013, Fig. 2). In such cases we divided the source area into primary and secondary sources: the former category corresponds to the shallow translational slide and the latter to scoured areas within debris flow tracks. Secondary sources were also separated as seemingly fresh slides occurring inside shallow translational run-out regions.

\section{Method}

The methodology followed is schematically represented in Fig. 3. The pre-processing consisted of fusing the $0.5 \mathrm{~m}$ resolution panchromatic band with the lower-resolution RG-B-NIR bands and orthorectifying the GeoEye-1 postevent image, using the orthophotos and the lidar DTM, as described in Lira et al. (2013). The pan-sharpening band fusion was performed with the Gram-Schmidt method available in ENVI software (http://www.exelisvis.com/docs/ GramSchmidtSpectralSharpening.html). The procedure involved the co-registration of the image bands (even though no shift had been observed in the original channels) followed by interpolation and resampling with the nearest neighbor method. We have measured the error introduced by the pansharpening process using the spectral quality indices (varying between 0 and 1) computed with ENVI which ranged between 0.88 and 0.96 for the four pan-sharpened bands. Segmentation tests using co-registered stacks of the origi- 


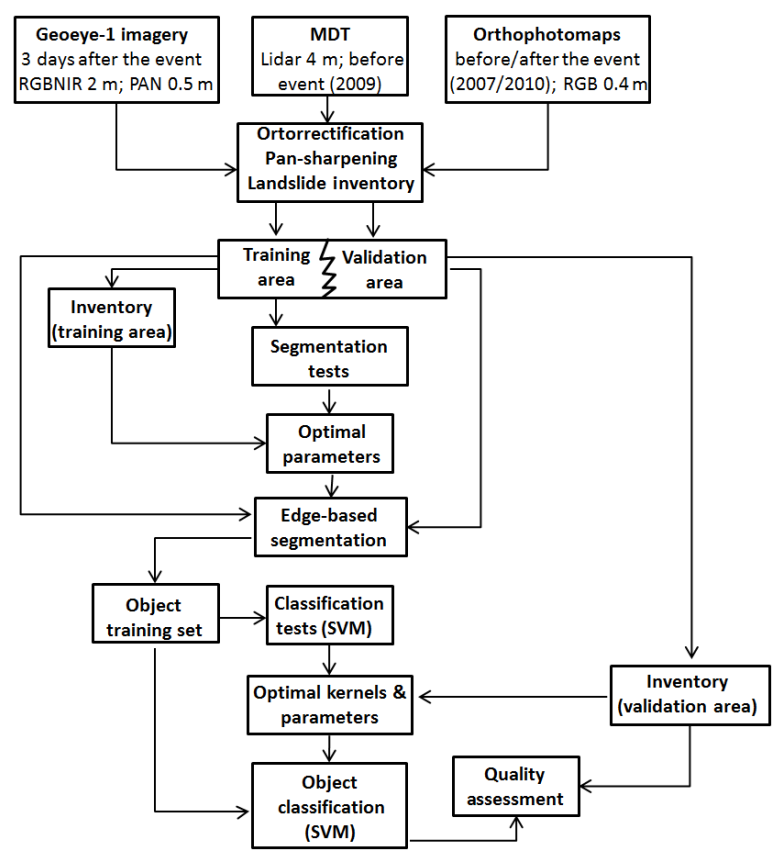

Figure 3. Diagram of the landslide mapping methodology.

nal bands were compared with segmentation using the pansharpened image, clearly indicating the superior performance of the latter, as expected from previous studies (e.g., Sarkar and Kanungo, 2004). The study area (with $15 \mathrm{~km}^{2}$, depicted in Figs. 1 and 5) was divided into a training area, from which the examples for learning were taken, and two smaller validation areas (totaling $3 \mathrm{~km}^{2}$ ), which did not contribute with examples for the training (see delimitation of training and validation areas in Fig. 5). The validation areas count a total of 85 landslides, comparing with about 115 used as examples in the training area. Inventory data belonging to the training area were used to select optimal segmentation parameters. Following segmentation/merging steps to delineate the structures or objects in the images, the classification into landslide/non-landslide or source/non-source classes was performed using a set of describing features of spectral, textural, and spatial nature.

\subsection{Segmentation}

The partition of the pan-sharpened and orthorectified GeoEye-1 image into objects was achieved with ENVI's feature extraction module, which performs image segmentation followed by merging of the segmented regions. Image segmentation involved computation of a gradient image for each of the (R, G, B, NIR) fused bands using a Sobel edge detection operator (Sobel, 1968), followed by conversion to a single-band map, where each pixel retains the maximum gradient across the bands. A watershed algorithm (Beucher and Lantuéjoul, 1979; Roerdink and Meijster, 2001) is then applied, flooding the map starting with the lowest gradient values (the most uniform part of the objects) to the highest gradient values (the edges). A selectable scale parameter can modify the gradient map, thus controlling the minimum contrast of the object edges (Roerdink and Meijster, 2001; Jin, 2012; http://www.exelisvis.com/docs/ BackgroundSegmentationAlgorithm.html). A higher value of this parameter diminishes the local sensitivity, which usually results in fewer and larger image objects. In the postsegmentation merging step, the spectral similarities of objects are evaluated and merged if their spectral properties are similar. To this effect, the mean spectral values for each band, in each adjacent object, and their Euclidean distance are computed, using the full lambda schedule method (Robinson et al., 2002). A selectable merge parameter modifies the spectral distance threshold below which two adjacent objects can be merged, hence controlling the homogeneity of the pixels within the final segmented regions (http://www.exelisvis. com/docs/BackgroundMergeAlgorithms.html).

Our goals in the segmentation/merging procedure were, first, to outline the landslides source and run-out areas, including the smallest of them, with as much accuracy as possible; and second to avoid the division of these features into many objects, so as not to obscure its recognizable landslide shape. In a sense, we searched for a balance between oversegmentation and under-segmentation - bearing in mind that the former is always preferable (Martha et al., 2010) - in an attempt to capture the geometric attributes of the landslide source and run-out areas (such as elongation or roundness) with minimum loss of accuracy in the delineation of contours. We tested several scale $(5,10,20,30,40,50,60$, and 70) and merge (30, 50, 70, 85, 90, and 95) parameters and superposed the final segmented regions onto the landslide inventory (solely in the training area) to find, by visual interpretation, the most appropriate combination to our case study (which turned out to be scale value 40 and merge value 90). Figure 4 shows details of the comparison between the inventory landslide delineation and our final choice for image partition. In the absence of an inventory map, the comparison can be made directly with the pan-sharpened RGB GeoEye-1 image also shown in the figure.

\subsection{Object features}

From (i) the four pan-sharpened bands of GeoEye-1, (ii) a calculated vegetation index (NDVI), and (iii) three topographic indexes derived from a $4 \mathrm{~m}$ resolution DTM (Table 1), a set of describing features of spectral, textural, and spatial natures were computed for each object of the final partitioned image. In Table 1, "curvature profile" refers to the curvature of the surface in the direction of slope and "curvature plan" refers to the curvature of the surface perpendicular to the slope direction.

The information from the nine input layers in Table 1 was used to compute the full set of spectral, textural, and spatial features available on ENVI's feature extraction (as described 

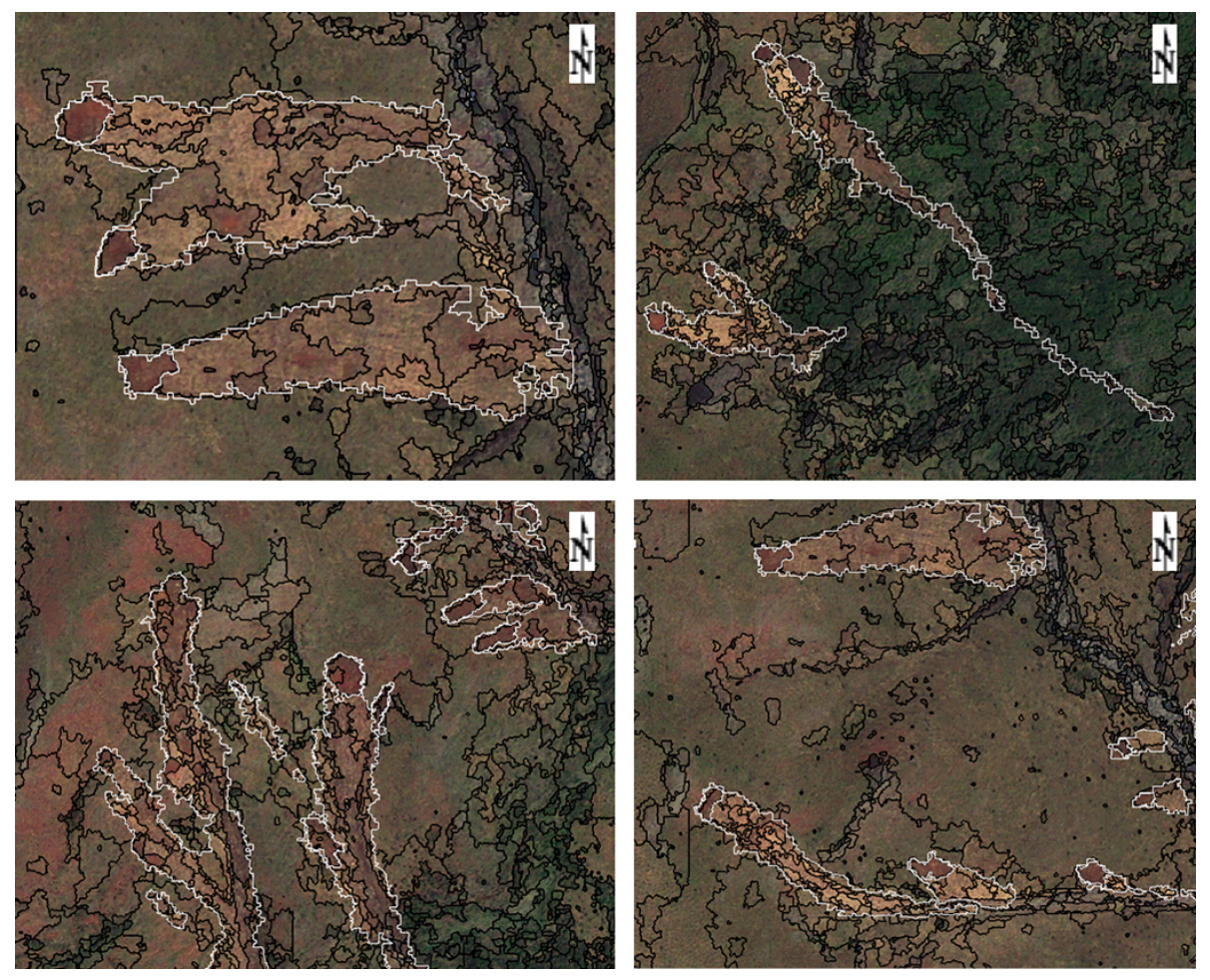

Figure 4. Details of GeoEye-1 pan-sharpened RGB image, showing the partition segments resulting from the chosen segmentation/merging parameters: scale 40, merge 90; white contour is the inventory landslide delineation; black contour is the image partition.

Table 1. Layers used in classification.

\begin{tabular}{lll}
\hline Spectral bands (GeoEye-1) & Vegetation index (GeoEye-1) & Topographic index (pre-event lidar DTM) \\
\hline RED $(50 \mathrm{~cm})$ & NDVI $(50 \mathrm{~cm})$ & Slope $(4 \mathrm{~m})$ \\
GREEN $(50 \mathrm{~cm})$ & & Aspect $(4 \mathrm{~m})$ \\
BLUE $(50 \mathrm{~cm})$ & & Curvature profile $(4 \mathrm{~m})$ \\
NIR $(50 \mathrm{~cm})$ & Curvature plan $(4 \mathrm{~m})$ \\
\hline
\end{tabular}

in www.exelisvis.com/docs/Example_Based_Classification. $\mathrm{html}$ ), resulting in 86 features for use in the object-based classification. We visually inspected the 86 color-coded object feature maps of the study area and excluded the noisiest ones, which resulted in 75 maps remaining maps corresponding to features that could bring some actual knowledge into the classification. In many cases, the feature maps were clear enough to allow identification of landscape signatures such as landslides, riverbeds, or slope changes. Training the SVM using either the selected group of features or the full set of features available led to very similar results, indicating low sensitivity of the algorithm to feature selection. We therefore chose to use all spectral, textural, and spatial object features available, as shown in Table 2.

\subsection{Supervised classification}

Classification was based on the SVM algorithm (Cortes and Vapnik, 1995; Vapnik, 1995), a supervised non-parametric statistical learning technique which separates the data set into groups or classes in a way consistent with the training examples. SVMs are gaining popularity in the remote sensing field, including landslide mapping (Van Den Eeckhaut et al., 2012; Moosavi et al., 2014), due to their ability to handle data with unknown statistical distributions and small training sets, as is often the case in this field (Mountrakis et al., 2011). SVMs are binary classifiers whose aim is to find the decision region boundary that separates the data set characteristics or features into two regions in the feature space. The SVM chooses the boundary (optimal hyperplane) with the maximum safety margin to the closest training features (termed support vectors), hence maximizing the margin between the classes. The linearization of the decision boundary 
Table 2. Features used for object-based learning.

\begin{tabular}{lll}
\hline Categories & Features & \\
\hline Spectral features & - Mean & $\begin{array}{l}\text { The spectral mean, SD, max, and min value } \\
\text { of the pixels inside the segmented region } \\
\text { are computed for each spectral band, } \\
\text { vegetation, and topographic indexes. }\end{array}$ \\
& - Maximum & A $3 \times 3$ moving window computes range, \\
Texture features & - Range & variance, and entropy of the pixels inside it, \\
& - Variance & followed by averaging inside the segmented region. \\
& - Entropy & - Elongation \\
Spatial features & - Area & - Major length \\
& - Length & - Minor length \\
& - Compactness & - Rectangular fit \\
& - Roundness & - Main direction \\
& - Form factor & - Number of holes \\
& - Convexity &
\end{tabular}

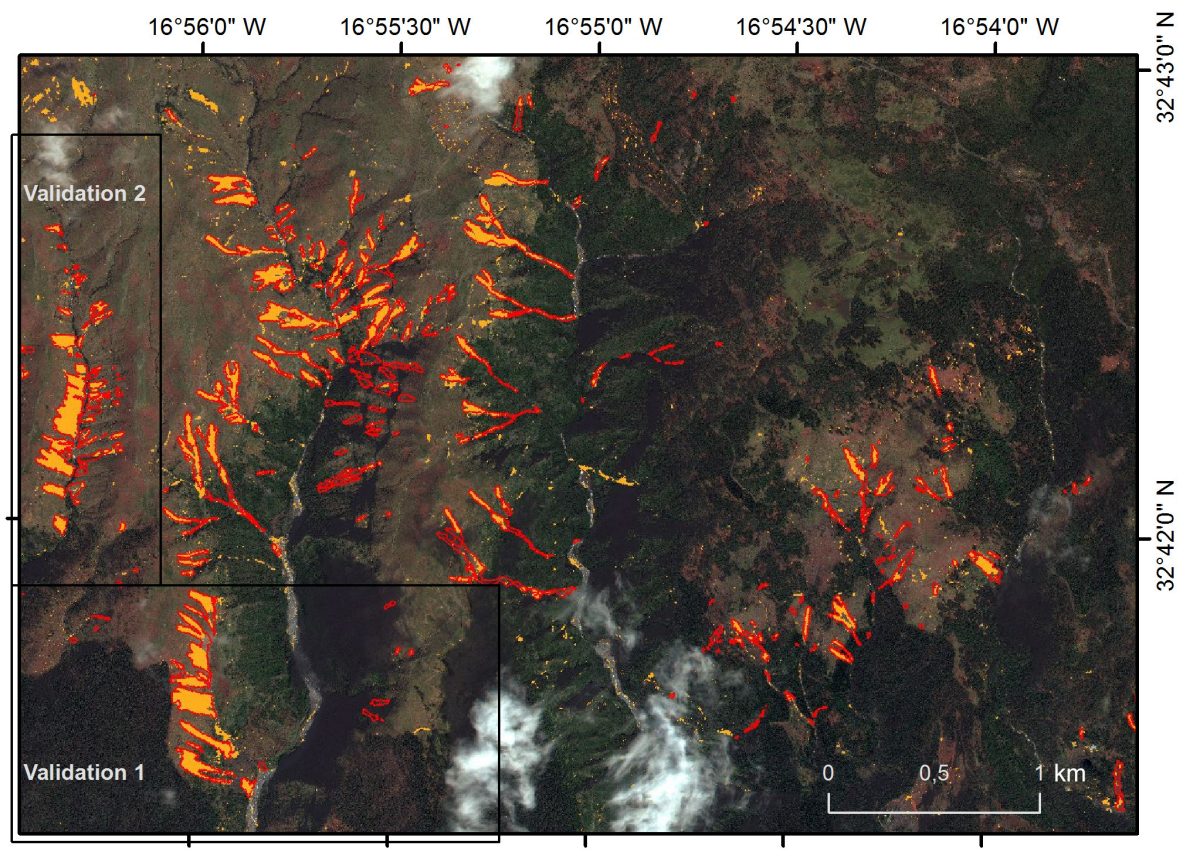

Figure 5. GeoEye-1 pan-sharpened RGB image over the study area $\left(15 \mathrm{~km}^{2}\right)$ showing the results of object-based SVM (RBF) landslide mapping. The area automatically classified as landslide (yellow fill) is compared to inventory reference data (red contours). Outlines of validation areas 1 and 2 also shown.

is achieved through the use of kernel functions which map the training data into a higher-dimensional space in which the two classes can be linearly separated by a hyperplane.

As referred before, the study area (with $15 \mathrm{~km}^{2}$, see Fig. 5) was divided into a training area, from which the examples for learning were taken, and two smaller validation areas (totaling $3 \mathrm{~km}^{2}$ ). Care was taken to ensure that the validation areas contained regions characterized by diverse land cover and solar illumination. Example objects in the training set were retrieved from the reference inventory map and represent a small percentage ( $1 \%$, about 1350 out of 130400$)$ of all the objects classified. Despite the larger dimensions of the training region, the number of landslides selected as training examples was similar to the amount of landslides appearing in the validation areas, in part due to a higher concentration of landslides in the latter. The choice of examples was expertdriven, aiming at sampling the diverse characteristics of each class.

We conducted several tests in order to choose the optimal SVM kernel function and set the corresponding parameters, namely the penalty parameter (which controls how examples located on the wrong side of the decision boundary are penal- 
ized) and the sigma function parameter (which defines the radius of influence of each training sample). Within the ENVI image processing environment, the SVM classifier was tested using different kernel functions (linear, polynomial (degrees 2-6), sigmoid, and radial basis function (RBF)). This was first done in an expedite manner, by visual inspection of the match between results and reference data in the training areas, and led to the choice of an RBF function with sigma value 0.03 and penalty parameter 100 . These tests were further refined, following Yang (2011), through a sensitivity analysis of the SVM parameters, using quantitative measures of the match between results and reference data in the validation areas. For RBF and degree 2 polynomial kernels, we exploited the range of sigma values between 0.01 and 0.30 , fixing the ENVI's penalty parameter at the default value of 100. Furthermore, for the RBF kernel we tested the SVM with penalty values in the range from 1 to 1000 and sigma values of $0.03,0.04$, and 0.2 . Finally, the SVM was tested for the degree 2 polynomial kernel, with sigma values of 0.03 and 0.04 and penalty values in the range from 100 to 250 . The best prediction accuracy was achieved with the RBF kernel, with sigma value 0.03 and penalty parameter 200, but the results varied very little with respect to those of our expedite test. The classification confidence threshold was set to $90 \%$, i.e., objects with less than $90 \%$ confidences in each class were set to unclassified.

To address our multi-class classification problem (landslide source; landslide run-out; all others) we started by using two "one-against-all approaches", in which a landslideagainst-all and a source-against-all classifier were designed to derive the classes "landslide", "landslide source", and "all others"; subsequently, the class "landslide run-out" was obtained from spatial subtraction of classes "landslide" and "landslide source". The feature information used by the SVM classifier was described in Sect. 3.2 and is listed in Tables 1 and 2 . The features associated with landslide/non-landslide examples or source/non-source examples are plotted by the algorithm into its corresponding feature spaces for definition of the decision boundaries. Then the entire image is classified: for each one-against-all approach, and for each object on the image, the SVM maps its spectral, spatial, and textural characteristics into the feature space and, depending on which side of the decision boundary it plots, classifies it accordingly. The set of features used by the "landslideagainst-all" classifier was the same employed by the "sourceagainst-all" classifier. However, from qualitative analysis of the feature maps produced for each of the (over 80) spectral, textural, and geometric attributes, we can state that spectral measures were the primary discriminant for source areas, followed by geometric attributes (e.g., elongation) and texture. The DTM used was acquired before the 2010 landslide event, so it does not represent surface changes occurred during it. Nevertheless, it provides unique geomorphic features (such as slope, aspect, and curvature) to each segmented object, assisting the classifier decision. The use of this information proved in our case very useful to diminish the ambiguity presented by objects with similar spectral characteristics located in flat areas.

\subsection{Accuracy assessment}

For assessment of the accuracy of the match between the classified image and the reference data, we compared the classification results in the validation areas with the landslide inventory map (Lahousse et al., 2001; Holbling et al., 2012) and followed standard metrics derived from the error or confusion matrix built from the two data sets (Congalton, 1991; Foody, 2002). In assessing the accuracy of results, we have separated the landslide/not-landslide classification from the source/run-out/not-landslide classification. In the latter case, we weighted equally the three possible classification errors or, in order words, we did not consider a lesser mistake to misclassify as source or run-out an object belonging to a landslide, instead of giving that same label to an object outside the landslide. The accuracy metrics computed were the overall accuracy (acc), which gives the proportion of area correctly classified, expressed as a percentage; the kappa index of agreement $(k)$, a measure of reliability which gives the proportion of agreement corrected for chance between two cases; and the omission (false negatives) and commission (false positive) errors. It is important to note that the overall accuracy is not a useful metric to evaluate classifiers in applications with imbalanced class distributions (i.e., when the number of samples in different classes varies greatly) such as in our case and in many other real-world applications ( $\mathrm{He}$ et al., 2009). Since the overall accuracy gives us the percentage of pixels correctly classified, the result is that, with imbalanced data sets, the performance of the classifier on the class of interest (e.g., landslide) will have much less effect on the overall accuracy than the performance on the majority class (not-landslide). Because of this, when discussing our accuracy results we use the false positive rates (FPRs) and false negative rates (FNRs) of the class of interest (landslide or source, depending on the one-against-all approach). The overall accuracy values appear solely in Table 3 for completion reasons.

\section{Results}

Following the methodology already described, landslide classification maps were produced for the $15 \mathrm{~km}^{2}$ wide study area located within one of the regions most affected by the 2010 landslide event on Madeira. In this section we present, evaluate, and discuss the results, which are divided into two parts: (i) landslide recognition and delineation and (ii) internal mapping of source and run-out areas. 
Table 3. Accuracy measures for landslide recognition (landslide-against-all classifier) and separation of landslide source and run-out (sourceagainst-all classifier), for different validation regions (VAL 1 and VAL 2). Two cases are distinguished, depending on whether reference data source areas are primary sources only or include secondary sources (seemingly fresh slides occurring inside the run-out area).

\begin{tabular}{lllll|lllll|llll}
\hline & \multicolumn{4}{c}{ Landslides } & \multicolumn{4}{c}{ Primary sources } & \multicolumn{3}{c}{ Primary and secondary sources } \\
\cline { 2 - 12 } & acc & $k$ & FNR & FPR & acc & $k$ & FNR & FPR & acc & $k$ & FNR & FPR \\
\hline VAL 1 & 98.2 & 0.80 & 17.6 & 19.9 & 98.4 & 0.34 & 24.9 & 77.2 & 98.4 & 0.48 & 36.7 & 60.3 \\
VAL 2 & 96.1 & 0.73 & 25.6 & 23.8 & 97.6 & 0.48 & 18.9 & 64.7 & 97.4 & 0.58 & 37.5 & 44.1 \\
\hline
\end{tabular}

acc: overall accuracy $(\%) ; k$ : Kappa index; FNR: false negative rate $(\%)$; FPR: false positive rate $(\%)$.
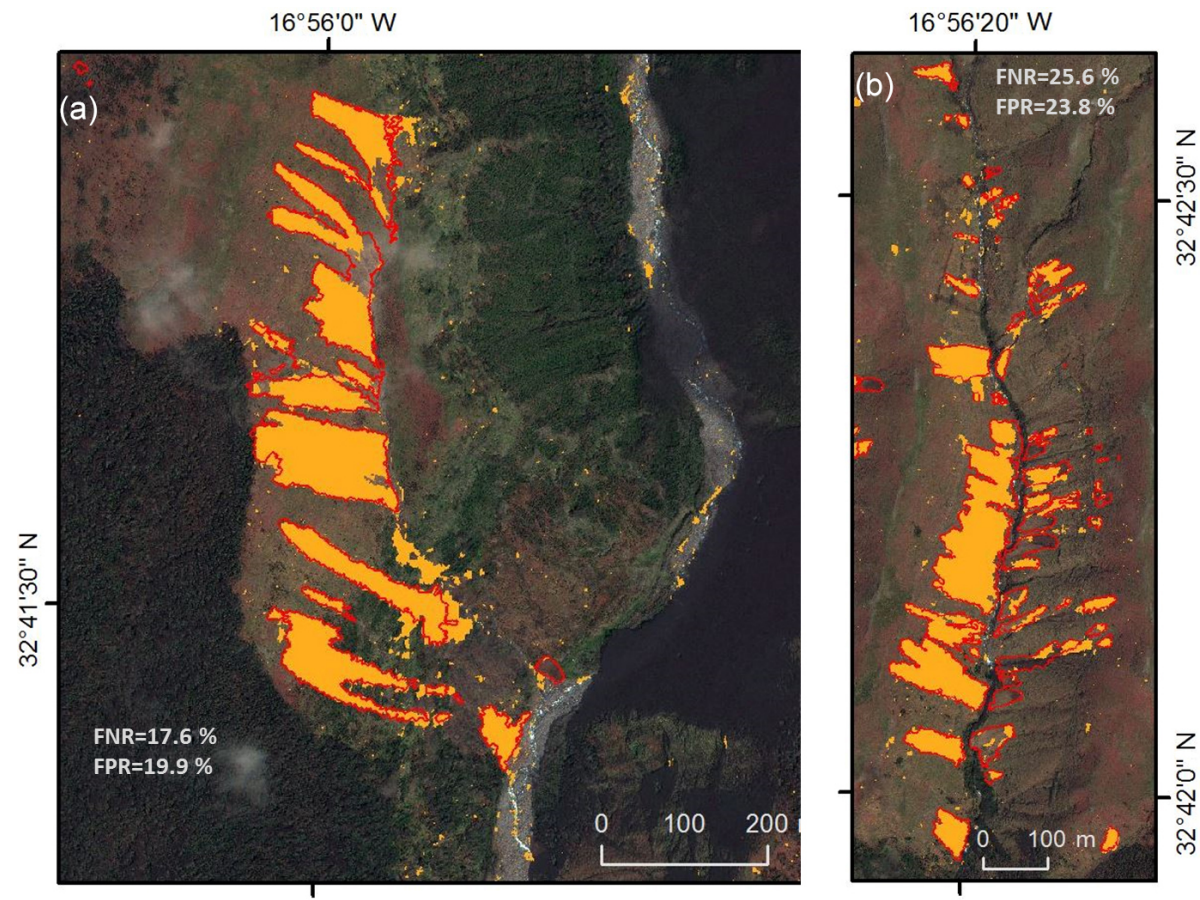

Figure 6. Details of the previous figure, showing the comparison between classified landslides (yellow fill) and inventory reference data (red contours) in the validation regions 1 (a) and 2 (b). FPR (or commission error) gives the ratio of the number of pixels incorrectly classified as landslide by the total number of pixels in the landslide class. FNR (or omission error) gives the ratio of the number of pixels actually belonging to a landslide but missed by the classifier, by the total number of pixels in the landslide inventory.

\subsection{Landslide recognition}

Figure 5 presents, for the overall study area, the object-based image classification of landslides, using the SVM machinelearning algorithm with the RBF kernel, as described before. To illustrate the performance of the approach, the semiautomatically recognized landslides are compared, in terms of overlapping area, to the inventory reference data (yellow fill and red contours in Fig. 5, respectively). We observe a remarkably accurate semiautomated depiction of the landslide areas, both in the training and validation regions. Landslides not successfully detected are located in areas obscured by shadows, an unavoidable hindrance in this approach.

In Fig. 6 the detailed mapping of the landslide areas is presented for the validation regions 1 and 2, which did not contribute with examples for learning. The figure also summarizes the accuracy metrics computed from the error matrix built for each of the validation areas, yielding good results for both of them, with commission errors below $26 \%$ and omission errors below $24 \%$ (see also Table 3 ). Note that we did not try to apply post-processing filters to exclude very small objects falsely identified as landslides but such a procedure would have reduced the commission errors.

Validation area 2, which contains a poorly illuminated slope, displays somewhat poorer accuracy in the classification of the overall area affected by a landslide. However, this problem is overcome by the source-against-all classification results (next Sect. 4.2), which performed well in what concerns landslide recognition: 61 out of 63 landslides were detected in this region, which compares with 20 out of 22 land- 

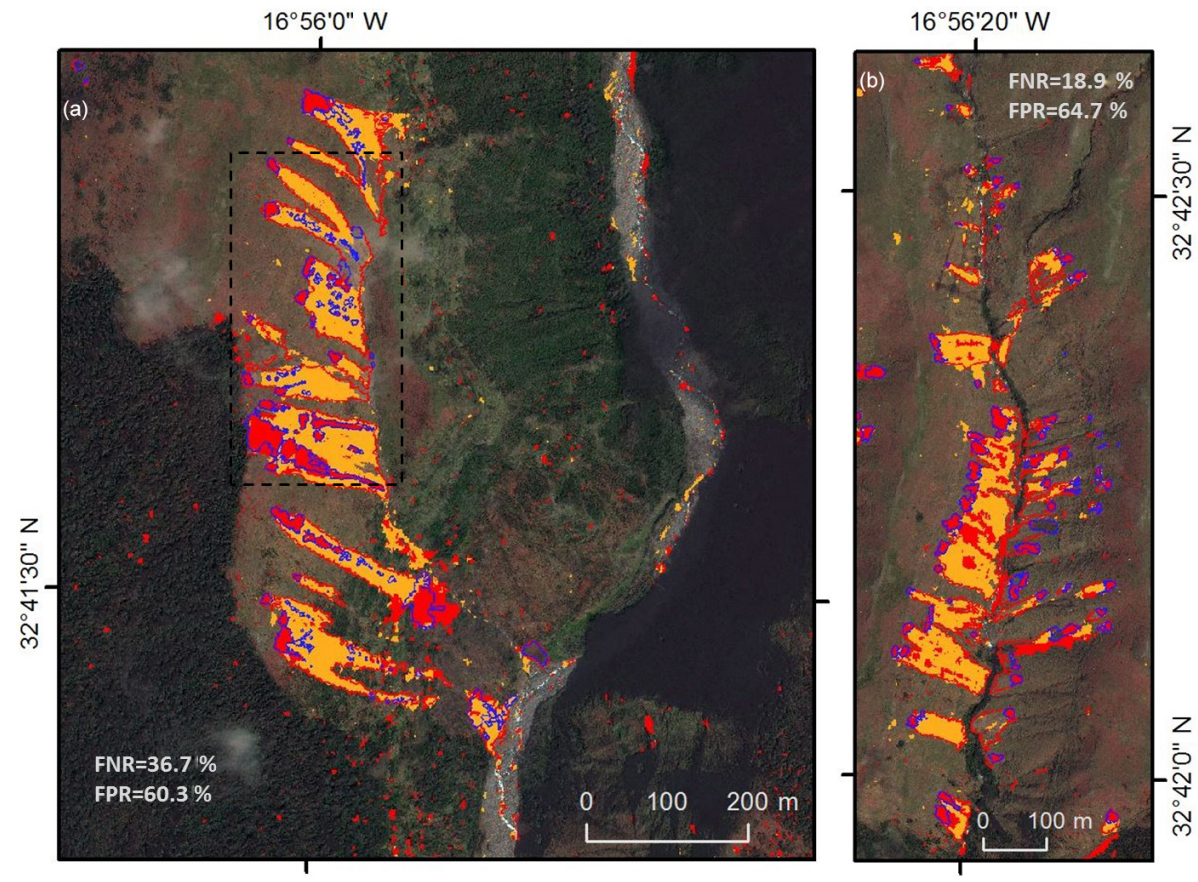

Figure 7. Classified landslide sources (red fill) and run-out (yellow fill) compared to inventory reference data (red contours delineate the landslide; blue contours delineate the source area). In the examples shown, source areas are defined either as primary sources only (in b, validation region 2) or also including secondary sources of sediment (in a, validation region 1). GeoEye-1 pan-sharpened RGB composite as base image. The area inside the dashed outline corresponds to Fig. 8.

slides in validation region 1 (see Fig. 7a and b). These values correspond to the detection of $95 \%$ of the landslides scars in the validation areas.

\subsection{Separation of source and run-out areas}

Figure 7 displays the results of the semiautomated mapping of landslide source and landslide run-out (transport plus deposition) in the validation areas 1 and 2, using again the object-based and SVM machine-learning approaches described in Sect. 3.

In Fig. 8 a detail of the classification is shown to allow comparison with the landslide characteristics that can be visually recognized in the pan-sharpened GeoEye image.

The inspection of Figs. 7 and 8 shows a good performance of the classifier in the internal mapping of source and run-out landslide areas, particularly in the sunnier east-facing slopes. In the less illuminated areas the classifier is able to map the source areas accurately but performs poorly in what concerns the landslide run-out mapping (Fig. 7). Accuracy measures were again computed for each of the validation areas, by comparison with source and run-out areas in reference data. Two cases need to be distinguished, depending on whether reference data source areas are primary sources only (example in Fig. 7b) or include secondary sources (seemingly fresh slides occurring inside the run-out region; Figs. 7a and 8).
Table 3 lists the computed overall accuracies, kappa indexes, and FNRs/FPRs for both validation areas and for both definitions of source area. FNR and FPR values fall below 38 and $78 \%$, respectively, for all situations (the results in Fig. 7 correspond to the best cases). The overall accuracy seems hindered, on the one hand, by the difficulties of the classifier in mapping the run-outs in poorly illuminated areas and, on the other hand, by the subjectivity of reference data description as primary or secondary sources of sediment.

\section{Conclusions}

We present a method for semiautomated landslide recognition and mapping of landslide source and run-out area, suitable for VHR remote sensing images of rain-induced landslide events. The approach combines object-based image analysis and an SVM supervised learning algorithm, and it was tested with a GeoEye- 1 multispectral image $(0.5 \mathrm{~m}$ panchromatic band), sensed 3 days after widespread landslides and flash floods on Madeira Island, using as ancillary data a pre-event high-resolution (4 m) lidar DTM. Our study confirms the high suitability of VHR multispectral images for landslide mapping (Van Westen et al., 2008), in particular with semiautomated methods, expanding the number of applications that target single post-event optical images (e.g., Cheng et al., 2013; Moosavi et al., 2014). In our study area, this choice proved much less demanding in terms of pre- 

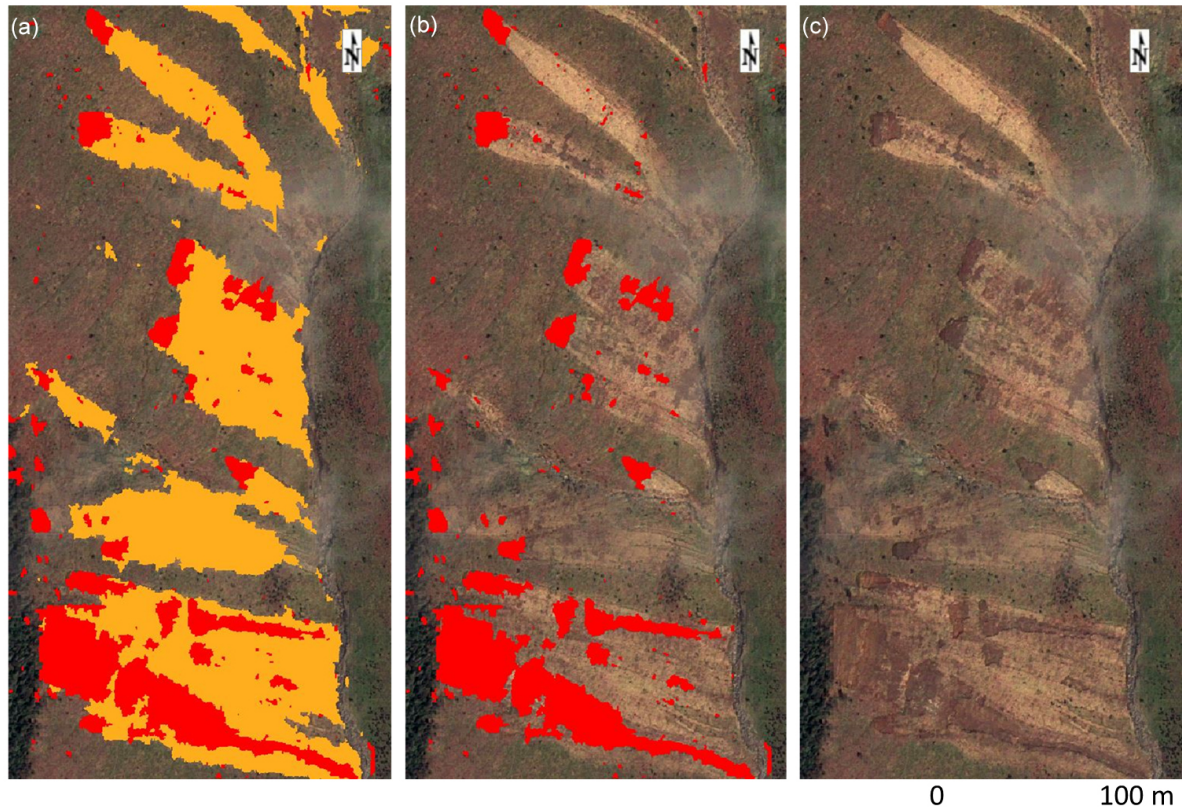

Figure 8. Detail of classification of landslide sources (red fill, a and b) and run-out (yellow fill, in a compared with base image c). GeoEye-1 pan-sharpened RGB composite as base image, whose area corresponds to the rectangular outline shown in Fig. 7.

processing than the change detection approaches more common in the literature (e.g., Lu et al., 2011; Mondini et al., 2011a, b), for which it is especially important to pay attention to co-registration and radiometric correction (Guzzetti et al., 2012). Our results also broaden the growing number of applications of object-based image analysis (OBIA) for automated landslide mapping, exemplifying the advantage of also using spatial and textural features in the classification and not only spectral attributes as in the pixel-based approach. Very few previous landslide studies have integrated OBIA and SVMs (Van den Eeckhaut et al., 2012; Moosavi et al., 2014), and we confirm that this is a robust and efficient approach, able to detect $95 \%$ of the number of landslides scars present in the validation areas. Also, to our knowledge, very few previous studies have used OBIA to tackle the problem of automated mapping of landslide source and run-out areas in optical images: Holbling et al. (2015) have recently tested such an approach in northern Taiwan, but their reference data set excluded debris flows or other sediment transport areas. In contrast, Mondini et al. (2011a, 2013) developed semiautomated pixel-based approaches to map landslide source and run-out areas, and our OBIA results compare well with theirs in terms of match to reference data.

At present, one of the main limitations of the proposed methodology is its poor performance in the automated mapping of landslide transport and deposition areas in poorly illuminated slopes, a problem that may perhaps be overcome using multiple satellite acquisition geometries. Another source of uncertainty results from the subjectivity associated with the definition of the landslide source areas, made mostly from visual inspection of post-event satellite images and orthophotos. These difficulties may have constrained the quantitative assessment of the classifier results. This task was particularly challenging in the case of debris flows, in which the high-energy transport area seemed to contain secondary sources of sediment supply (see examples in Fig. 8), with the same spectral and textural image characteristics of the primary sources. Another limitation is the subjectivity of the trial-and-error procedure used to select the segmentation parameters. Such expert-driven approach was used to minimize over-segmentation, in order to capture the geometrical attributes of the landslides which proved to be relevant in the classification, but we cannot exclude that automated methods for objective determination of segmentation parameters (see, e.g., Dragut et al., 2010, 2014; Martha et al., 2011; Gao et al., 2011) would yield better results. In a similar way, the use of objective automated feature selection methods (e.g., Stumpf and Kerle, 2011) could further improve the effectiveness of our method or the accuracy of the results.

The method proposed here may have the potential to increase promptness and cost effectiveness in the production of inventories following a landslide event, when a VHR postevent optical image and a pre-event digital elevation model are both available. It also assists an approximate spatial quantification of the amount of sediments produced and transported during a landslide event, information that can be crucial in emergency response situations, and is clearly important for landslide susceptibility and hazard assessment (Guzzetti et al., 2009), contributing in particular to support 
post-event mitigation actions, such as sediment control measures (Lira et al., 2013).

Acknowledgements. This contribution was developed in the frame of the project AULIS (PTDC/ECM/116611/2010) funded by Fundação para a Ciência e a Tecnologia (FCT) in Portugal. Sandra Heleno acknowledges FCT for her research grant SFRH/BPD/84796/2012.

Edited by: P. Reichenbach

Reviewed by: three anonymous referees

\section{References}

Aksoy, B. and Ercanoglu, M.: Landslide identification and classification by object-based image analysis and fuzzy logic: an example from the Azdavay region (Kastamonu, Turkey), Comput. Geosci., 38, 87-98, 2012.

Baioni, D.: Human activity and damaging landslides and floods on Madeira Island, Nat. Hazards Earth Syst. Sci., 11, 3035-3046, doi:10.5194/nhess-11-3035-2011, 2011.

Barlow, J., Franklin, S., and Martin, Y.: High spatial resolution satellite imagery, DEM derivatives, and image segmentation for the detection of mass wasting processes, Photogramm. Eng. Rem. S., 72, 687-692, 2006.

Beucher, S. and Lantuejoul, C.: Use of watersheds in contour detection, International Workshop on Image Processing, Real-Time Edge and Motion Detection/Estimation, Rennes, France, 17-21 September 1979, 2.1-2.12, 1979.

Borghuis, A. M., Chang, K., and Lee, H. Y.: Comparison between automated and manual mapping of typhoon-triggered landslides from SPOT-5 imagery, Int. J. Remote Sens., 28, 1843-1856, 2007.

Bucknam, R. C., Coe, J. A., Chavarría, M. M., Godt, J. W., Tarr, A. C., Bradley, L.-A., Rafferty, S., Hancock, D., Dart, R. L., and Johnson, M. L.: Landslides Triggered by Hurricane Mitch in Guatemala - Inventory and Discussion, USGS, Denver, Colorado, Open-File Report, 443 pp., 2001.

Chang, Y.-L., Liang, L.-S., Han, C.-C., Fang, J.-P., Liang, W.Y., and Chen, K.-S.: Multisource data fusion for landslide classification using generalized positive Boolean functions, IEEE T. Geosci. Remote, 45, 1697-1708, doi:10.1109/TGRS.2007.895832, 2007.

Cheng, G., Guo, L., Zhao, T., Han, J., Li, H., and Fang, J.: Automatic landslide detection from remote-sensing imagery using a scene classification method based on BoVW and pLSA, Int. J. Remote Sens., 34, 45-59, 2013.

Chini, M., Cinti, F. R., and Stramondo, S.: Co-seismic surface effects from very high resolution panchromatic images: the case of the 2005 Kashmir (Pakistan) earthquake, Nat. Hazards Earth Syst. Sci., 11, 931-943, doi:10.5194/nhess-11-931-2011, 2011.

Congalton, R. G.: A review of assessing the accuracy of classifications of remotely sensed data, Remote Sens. Environ., 37, 35-46, 1991.

Cortes, C. and Vapnik, V.: Support-vector networks, Mach. Learn., 20, 273-297, 1995.
Couto, F. T., Salgado, R., and Costa, M. J.: Analysis of intense rainfall events on Madeira Island during the 2009/2010 winter, Nat. Hazards Earth Syst. Sci., 12, 2225-2240, doi:10.5194/nhess-122225-2012, 2012.

Cruden, D. M. and Varnes, D. J.: Landslide types and processes, in: Landslides, Investigation and Mitigation, Transportation Research Board, edited by: Turner, A. K. and Shuster, R. L., Special Report 247, National Academy Press, Washington DC, 36-75, 1996.

Danneels, G., Pirard, E., and Havenith, H. B.: Automatic landslide detection from remote sensing images using supervised classification methods, in: Proceedings of IGARSS 2007 - IEEE International Geoscience and Remote Sensing Symposium, 23-28 July 2007, Barcelona, 3014-3017, 2007.

Dragut, L., Tiede, D. and Levick, S.: ESP: a tool to estimate scale parameter for multiresolution image segmentation of remotely sensed data, Int. J. Geogr. Inf. Sci., 24, 859-871, 2010.

Dragut, L., Csillik, O., Eisank, C. and Tiede, D.: Automated parametrisation for multi-scale image segmentation on multiple layers, Int. Soc. Photogramme, 88, 119-127, 2014.

Fragoso, M., Trigo, R. M., Pinto, J. G., Lopes, S., Lopes, A., Ulbrich, S., and Magro, C.: The 20 February 2010 Madeira flashfloods: synoptic analysis and extreme rainfall assessment, Nat. Hazards Earth Syst. Sci., 12, 715-730, doi:10.5194/nhess-12715-2012, 2012.

Foody, G. M.: Status of land cover classification accuracy assessment, Remote Sens. Environ., 80, 185-201, 2002.

Gabet, E. and Mudd, S.: The mobilization of debris flows from shallow landslides, Geomorphology, 74, 207-218, 2006.

Gao, Y., Mas, J. P., Kerle, N., and Pacheco, J. A.: Optimal region growing segmentation and its effect on classification accuracy, Int. J. Remote Sens., 32, 3747-3763, 2011.

Guzzetti, F., Ardizzone, F., Cardinali, M., Rossi, M., and Valigi, D.: Landslide volumes and landslide mobilization rates in Umbria, central Italy, Earth Planet. Sc. Lett., 279, 222-229, 2009.

Guzzetti, F., Mondini, A., Cardinali, M., Fiorucci, F., Santangelo, M., and Chang, K.: Landslide inventory maps: new tools for an old problem, Earth-Sci. Rev., 112, 42-66, 2012.

He, H. and Garcia, E. A.: Learning from Imbalanced Data, IEEE Trans. Knowl. Data Eng., 21, 1263-1284, 2009.

Heleno, S., Matias, M., Vasconcelos, M., Sousa, J., and Pina, P.: Automated OBIA mapping and classification of rain-induced landslides with VHR post-event optical images and HR pre-event LiDAR DEM, South-Eastern European Journal of Earth Observation and Geomatics, 3, 271-274, 2014.

Holbling, D., Füreder, P., Antolini, F., Cigna, F., Casagli, N., and Lang, S.: A semi-automated object-based approach for landslide detection validated by persistent scatterer interferometry measures and landslide inventories, Remote Sens., 4, 1310-1336, 2012.

Holbling, D., Friedl, B. and Eisank, C.: An object-based approach for semi-automated landslide change detection and attribution of changes to landslide classes in northern Taiwan, Earth Sci. Inform., 8, 327-335, 2015.

IPMA: Boletim Climatológico Mensal da Madeira - Setembro 2010, available at: http://www.ipma.pt/resources. www/docs/im.publicacoes/edicoes.online/20101011/ AXSDhzwOAugQKWqSDMhu/cli_20100901_20100930_ pcl_mm_md_pt.pdf (last access: January 2016), 2010. 
Jin, X.: Segmentation-Based Image Processing System, US Patent 8,260,048, filed 14 November 2007, issued 4 September 2012.

Joyce, K. E.,Dellow, G. D., and Glassey, P. J.: Assessing image processing techniques for mapping landslides, in: Proceedings of IGARSS 2008 - IEEE International Geoscience and Remote Sensing Symposium, 7-11 July 2008, Boston, MA, 1231-1234, 2008.

Kurtz, C., Stumpf, A., Malet, J., Gançarski, P., Puissant, A., and Passat, N.: Hierarchical extraction of landslides from multiresolution remotely sensed optical images, ISPRS J. Photogramm., 87, 122-136, 2014.

Lacroix, P., Zavala, B., Berthier, E., and Audin, L.: Supervised method of landslide inventory using panchromatic SPOT5 images and application to the earthquake-triggered landslides of Pisco (Peru, 2007, $M_{\mathrm{W}}$ 8.0), Remote Sens., 5, 2590-2616, 2013.

Lahousse, T., Chang, K. T., and Lin, Y. H.: Landslide mapping with multi-scale object-based image analysis - a case study in the Baichi watershed, Taiwan, Nat. Hazards Earth Syst. Sci., 11, 2715-2726, doi:10.5194/nhess-11-2715-2011, 2011.

Li, Y., Chen, G., Wang, B., Zheng, L., Zhang, Y., and Tang, C.: A new approach of combining aerial photography with satellite imagery for landslide detection, Nat. Hazards, 66, 649-669, 2013.

Lira, C., Lousada, M., Falcão, A. P., Gonçalves, A. B., Heleno, S., Matias, M., Pereira, M. J., Pina, P., Sousa, A. J., Oliveira, R., and Almeida, A. B.: The 20 February 2010 Madeira Island flashfloods: VHR satellite imagery processing in support of landslide inventory and sediment budget assessment, Nat. Hazards Earth Syst. Sci., 13, 709-719, doi:10.5194/nhess-13-709-2013, 2013.

Lu, P., Stumpf, A., Kerle, N., and Casagli, N.: Object-oriented change detection for landslide rapid mapping, IEEE Geosci. Remote S., 8, 701-705, 2011.

Luna, T., Rocha, A., Carvalho, A. C., Ferreira, J. A., and Sousa, J.: Modelling the extreme precipitation event over Madeira Island on 20 February 2010, Nat. Hazards Earth Syst. Sci., 11, 24372452, doi:10.5194/nhess-11-2437-2011, 2011.

Malamud, B. D., Turcotte, D. L., Guzzetti, F., and Reichenbach, P.: Landslide inventories and their statistical properties, Earth Surf. Proc. Land., 29, 687-711, 2004.

Marc, O. and Hovius, N.: Amalgamation in landslide maps: effects and automatic detection, Nat. Hazards Earth Syst. Sci., 15, 723733, doi:10.5194/nhess-15-723-2015, 2015.

Martha, T. R. and Kumar, K. V.: September 2012 landslide events in Okhimath, India - an assessment of landslide consequences using very high resolution satellite data, Landslides, 10, 469479, 2013.

Martha, T. R., Kerle, N., Jetten, V., van Westen, C. J., and Kumar, K. V.: Characterising spectral, spatial and morphometric properties of landslides for semi-automatic detection using object-oriented methods, Geomorphology, 116, 24-36, 2010.

Martha, T. R., Kerle, N., van Westen, C. J., Jetten, V., and Kumar, K. V.: Segment optimization and data-driven thresholding for knowledge-based landslide detection by object-based image analysis, IEEE T. Geosci. Remote, 49, 4928-4943, 2011.

Martha, T. R., Kerle, N., van Westen, C. J., Jetten, V., and Kumar, K. V.: Object-oriented analysis of multi-temporal panchromatic images for creation of historical landslide inventories, ISPRS J. Photogramm., 67, 105-119, 2012.
Mondini, A., Chang, K., and Yin, H.: Combining multiple change detection indices for mapping landslides triggered by typhoons, Geomorphology, 134, 440-451, 2011a.

Mondini, A., Guzzetti, F., Reichenbach, P., Rossi, M., Cardinali, M., and Ardizzone, F.: Semi-automatic recognition and mapping of rainfall induced shallow landslides using optical satellite images, Remote Sens. Environ., 115, 1743-1757, 2011 b.

Mondini, A. C., Marchesini, I., Rossi, M., Chang, K.-T., Pasquariello, G., and Guzzetti, F.: Bayesian framework for mapping and classifying shallow landslides exploiting remote sensing and topographic data, Geomorphology, 201, 135-147, 2013.

Mondini, A. C., Viero, A., Cavalli, M., Marchi, L., Herrera, G., and Guzzetti, F.: Comparison of event landslide inventories: the Pogliaschina catchment test case, Italy, Nat. Hazards Earth Syst. Sci., 14, 1749-1759, doi:10.5194/nhess-14-1749-2014, 2014.

Moosavi, V., Talebi, A., and Shirmohammadi, B.: Producing a landslide inventory map using pixel-based and object-oriented approaches optimized by Taguchi method, Geomorphology, 204, 646-656, 2014.

Mountrakis, G., Im, J., and Ogole, C.: Support vector machines in remote sensing: a review, ISPRS J. Photogramm., 66, 247-259, 2011.

Nichol, J. and Wong, M. S.: Satellite remote sensing for detailed landslide inventories using change detection and image fusion, Int. J. Remote Sens., 26, 1913-1926, 2005.

Pisani, R., Riedel, P., Costa, K., Nakamura, R., Pereira, C., Rosa, G., and Papa, J.: Automatic landslide recognition through optimumpath forest, in: Proceedings of IGARSS 2012 - IEEE International Geoscience and Remote Sensing Symposium, 22-27 July 2012, Munich, 6228-6231, 2012.

Pollard, T. B., Eden, I., Mundy, J. L., and Cooper, D. B.: A volumetric approach to change detection in satellite images, Photogramm. Eng. Rem. S., 76, 817-831, 2010.

Robinson, D. J., Redding, N. J., and Crisp, D. J.: Implementation of a Fast Algorithm For Segmenting SAR Imagery, Scientific and Technical Report, Defense Science and Technology Organization, Australia, 2002.

Roerdink, J. B. T. M. and Meijster, A.: The watershed transform: definitions, algorithms, and parallelization strategies, Fund. Inform., 41, 187-228, 2001.

Sarkar, S. and Kanungo, D. P.: An integrated approach for landslide susceptibility mapping using remote sensing and GIS, Photogramm. Eng. Rem. S., 70, 617-625, 2004.

Schürch, P., Densmore, A. L., Rosser, N. J. and McArdell, B. W: Dynamic controls on erosion and deposition on debris-flow fans, Geology, 39, 827-830, 2011.

Sidle, R. C. and Ochiai, H.: Landslides: processes, prediction, and land use, in: Water Resources Monograph, 18, American Geophysical Union, Washington, DC, 1-312, 2006.

Sobel, I.: An isotropic $3 \times 3$ image gradient operator, Presentation at Stanford Artificial Intelligence Project (SAIL), Stanford, California, doi:10.13140/RG.2.1.1912.4965, 1968.

Stumpf, A. and Kerle, N.: Object-oriented mapping of landslides using Random Forests, Remote Sens. Environ., 115, 2564-2577, 2011.

Stumpf, A., Lachiche, N., Malet, J.P., Kerle, N., and Puissant, A.: Active learning in the spatial domain for remote sensing image classification, IEEE Trans. Geosci. Remote Sens., 52, 2492 2507, 2014. 
Teixeira, J. C., Carvalho, A. C., Carvalho, M. J., Luna, T., and Rocha, A.: Sensitivity of the WRF model to the lower boundary in an extreme precipitation event - Madeira island case study, Nat. Hazards Earth Syst. Sci., 14, 2009-2025, doi:10.5194/nhess-14-2009-2014, 2014.

Theule, J., Liébault, F., and Laigle, D.: Spatial Variability of Channel Erosion by Debris-Flows (Field Observations in the French Alps), Eng. Geol. Soc. Territory, 3, 97-101, Springer, 2014.

Tiranti, D. and Deangeli, C.: Modeling of debris flow depositional patterns according to the catchment and sediment source area characteristics, Front. Earth Sci., 3, 1-14, 2015.

Van Den Eeckhaut, M. V. D., Kerle, N., Poesen, J., and Hervás, J.: Object-oriented identification of forested landslides with derivatives of single pulse LiDAR data, Geomorphology, 173-174, 3042, 2012.

Van Westen, C. J., van Asch, T. W. J., and Soeters, R.: Landslide hazard and risk zonation - why is it still so difficult?, B. Eng. Geol. Environ., 65, 167-184, 2006.

Van Westen, C. J., Castellanos, E., and Kuriakose, S. L.: Spatial data for landslide susceptibility, hazard, and vulnerability assessment: an overview, Eng. Geol., 102, 112-131, 2008.
Vapnik, V. N.: The Nature of Statistical Learning Theory, SpringerVerlag, Berlin, Germany, 1995.

Varnes, D. J.: Slope movement types and processes, in: Landslides Analysis and Control, edited by: Schuster, R. L. and Krizek, R. J., Special Report 176, Transportation Research Board, National Academy of Science, Washington DC, 11-33, 1978.

$\mathrm{Xu}, \mathrm{C} ., \mathrm{Xu}, \mathrm{X}$., Bruce, J., and Shyu, H.: Database and spatial distribution of landslides triggered by the Lushan, China $M_{\mathrm{W}} 6.6$ earthquake of 20 April 2013, Geomorphology, 248, 77-92, doi:10.1016/j.geomorph.2015.07.002, 2015.

Yang, X.: Parameterizing support vector machines for land cover classification, Photogramm. Eng. Rem. S., 77, 27-37, 2011.

Yang, X. and Chen, L.: Using multi-temporal remote sensor imagery to detect earthquake-triggered landslides, Int. J. Appl. Earth Obs., 12, 487-495, 2010.

Zêzere, J. L.: Landslide susceptibility assessment considering landslide typology. A case study in the area north of Lisbon (Portugal), Nat. Hazards Earth Syst. Sci., 2, 73-82, doi:10.5194/nhess2-73-2002, 2002. 